\title{
Use of a PLA Sleeve To Remove Electron Enhancement in Superficial X-Ray Therapy
}

Cathryn Barbagallo ( $\square$ cathryn.barbagallo@acpsem.org.au )

ACPSEM https://orcid.org/0000-0003-3462-9176

\section{Research Article}

Keywords: Superficial X-rays, Electron Enhanced Dose, 3D printing

Posted Date: November 10th, 2021

DOI: https://doi.org/10.21203/rs.3.rs-1022286/v1

License: () (i) This work is licensed under a Creative Commons Attribution 4.0 International License. Read Full License 


\section{Abstract}

A newly installed superficial x-ray unit was found to produce enhanced electron dose at the skin surface. ACPSEM guidelines suggest using nail varnish within the treatment cones as a method to reduce this dose. In this study, a 3D PLA sleeve was produced and used as an alternative to the nail varnish. Further, plastic wrap was also investigated for its anecdotal recommendations to reduce dose. It was found that a $1 \mathrm{~mm}$ printed sleeve, inserted into the treatment cone sufficiently reduced the enhanced dose to within approximately $3 \%$ of the dose measured with a Farmer-type chamber. The use of plastic wrap also reduced the enhanced dose, but impracticalities in its use make it non-viable for routine clinical use.

\section{Introduction}

The incidence of Non-Melanoma Skin Cancer (NMSC) in the Australian population is estimated to be around $2 \%$ and it continues to be an ongoing disease due to the nature of the climate and lifestyle (Perera et al., 2015). Whilst the use of Mohs Micrographic Surgery is considered the gold standard for treatment of skin cancers, the use of superficial x-rays for the treatment of skin cancers has proven to be the preferred treatment for many patients (McGregor et al., 2015). The major recipients of superficial x-ray treatment (SXRT) will be patients $>70$ years who may present with other co-morbidities. Further, many of the sites that may be difficult to treat with surgical means, are ideal candidates for radiotherapy. Dosimetry of kilovoltage beams is well established, but with new treatment machine design, new issues can present. This work is related to a newly released machine and the issue of electron surface dose enhancement.

In 2019 a new WOmed T105 SXRT machine (Wolf Medical, Germany) was installed in a standalone treatment clinic. As a standalone centre, they treat only with superficial energies with a high patient load. The newly installed machine was accepted, and commissioning was undertaken. As part of that process, the recommended testing, as per the ACPSEM recommendations were undertaken (Hill et al., 2018). The factory measurements for output at the end of the reference cone was compared with those undertaken during commissioning. While the work here was performed with a recommended reference chamber for output (Farmer-type), it was noted that electron enhancement at the surface maybe present due to the design of the cones.

The circular cones provided with the treatment unit were $1 \mathrm{~cm}, 2 \mathrm{~cm}, 3 \mathrm{~cm}$ and $4 \mathrm{~cm}$ open-ended stainless-steel cones at $15 \mathrm{~cm}$ SSD. In addition, there were two circular close-ended cones (Perspex); $5 \mathrm{~cm}$ at $15 \mathrm{~cm} \mathrm{SSD}$ and $10 \mathrm{~cm}$ at $30 \mathrm{~cm}$ SSD. All the reference measurements were performed using the $5 \mathrm{~cm}$ close-ended cone.

Electron dose enhancement is known consequence of the metal cones used for treatment. Close-ended cones, with a thin window of Perspex or other medium, provide sufficient shielding to remove this enhanced dose. Without the addition of an absorbing material the dose measured at the end of the cone and delivered to the patient can be enhanced significantly due to electrons depositing in the first few nm of skin.

Reference documents recommend that chambers used sufficiently absorb these electrons, and as such the Farmer chamber measurements are a true measure of dose due only to kilovoltage photons. Unfortunately, in some open cones the dose being received by the surface skin layers will have this enhanced dose present and could potentially have higher than expected dose to treatment volumes (Klevenhagen et al., 1991).

The Australasian recommendations to remove the electron dose is to coat the inner $10 \mathrm{~cm}$ of the cone with nail varnish (Hill et al., 2018). The acrylic of the nail varnish sufficiently absorbs the electrons produced. This does provide an inexpensive method to alleviate the issue but is not clear in how many coats are sufficient. Further, the nail varnish will degrade over time, requiring it to be reapplied and potentially being a hygiene hazard when the cones are positioned directly in skin contact.

Nail varnish is most commonly a nitrocellulose dissolved in a solvent like ethyl acetate. As the solvent evaporates, the resin in the nitrocellulose hardens forming a coating on the surface. Application of the nail varnish to the inner surface of the cones can be both time consuming and may give a non-uniform thickness throughout the cone. An alternative proposed here is to use a 3D printed sleeve inserted into the cone. 
3D printing is available within many clinical departments with the application including bolus for megavoltage treatment and to provide customised shielding in SXRT applications (Crowe et al., 2021). Further, many home users have been able to print small items using available resin. Online CAD software means that the design of and printing is now within reach of any user. Small commercial companies providing reliable printing services can offer a range of materials once the design has been sent directly to them.

Polylactic Acid (PLA) is the most used material in desktop extrusion printers as it requires a low heat to print, does not require a heated bed, and is relatively inexpensive. It has a physical density of $1.210-1.430 \mathrm{~g} / \mathrm{cm}^{3}$, and when printed in a thin layer is still relatively robust (Polyactic Acid, 2015). It was proposed that a sleeve of PLA could be inserted into the treatment cone as an alternative to the nail varnish method.

PLA has been assessed in its use for external beam radiotherapy (Van der Walt et al., 2019) and found to be a safe and effective material to use for electron and photon modalities for bolus. Other investigators have examined the use of PLA in 3D printing and the uncertainty and reproducibility in manufacture (Craft et al., 2018). In applications where the radiation beam will be passing through the material, the issues related the density and production are integral, but in this application, any minor variation in production will have no consequence. The work here examines the reduction in electron enhancement dose when using the PLA sleeve.

Anecdotally, the use of plastic wrap has been proposed to reduce the enhanced dose at the surface. Plastic wrap is most used in the food industry and home applications. It is generally a form of PolyVinyl Chloride but depending upon manufacturer could also be a low-density polyethylene. It is generally found in thicknesses in the range of 7.6-10 $\mu \mathrm{m}$ (Polyvinyl Chloride PVC: Properties, Benefits \& Applications, 2019). It was investigated here as another method of dose reduction that may be achievable in the clinic.

\section{Materials And Methods}

All tests were conducted on a WOmed T105 superficial x-ray therapy machine. The machine has four available treatment energies listed in the table below. Measurements were performed for the full range of energies, but the clinic most commonly treats using Filter No. 3.

Table 1: Available treatment energies on the WOmed T105 machine.

\begin{tabular}{|llll|}
\hline Filter No. & Acc. Voltage & Nominal HVL & Measured HVL \\
& $(\mathrm{kV})$ & & \\
\hline 1 & 55 & $0.4 \mathrm{mmAl}$ & $0.5 \mathrm{mmAl}$ \\
2 & 70 & $0.7 \mathrm{mmAl}$ & $1.1 \mathrm{mmAl}$ \\
3 & 100 & $1.0 \mathrm{mmAl}$ & $2.2 \mathrm{mmAl}$ \\
4 & 100 & $0.1 \mathrm{mmCu}+0.55 \mathrm{mmAl}$ & $3.9 \mathrm{mmAl}$ \\
\hline
\end{tabular}

The dose at the end of the reference cone $(5 \mathrm{~cm} / 15 \mathrm{~cm}$ SSD) was measured in absolute dose conditions as prescribed by the TG61 protocol in air, corrected for temperature and pressure (Ma et al., 2001). Although a range of cones was provided with the treatment machine, measurements here were limited to the $4 \mathrm{~cm}$ open cone and $5 \mathrm{~cm}$ close-ended cone. Smaller cones were not measured due to the relatively large size of the measurement chambers.

All measurements taken were delivered using 30 s readings, and readings shown here are the average recorded.

\section{Electron dose contribution}


To estimate the electron dose contribution, measurements were performed in two stages. In the first stage the output for the selected cones was measured in air using a FC-65P Farmer chamber (IBA Dosimetry GmBH, Germany). Filter 1 is below the recommended energy use for the Farmer but was included for comparison. The measured values derived a cone ratio or Relative Output Factor (ROF). The output was then repeated using a low energy thin window Exradin A11TW chamber (RPD Inc, USA) again for both cones. The ROF was then derived and a comparison of the value for each energy was used as a measure of the electron enhanced dose. Subsequent measurements were conducted with the Exradin chamber to determine direct reduction of electron dose. All measurements were conducted using the CNMC Model 10 electrometer (CNMC, USA).

\section{Dose Reduction Using Plastic Wrap}

Plastic wrap is commercially available and used in many clinics to wrap lead used for shielding. Wrapping the lead used aims to prevent toxicity to staff handling or patients with whom it may contact skin. A series of measurements were performed for one energy (Filter $2 / 70 \mathrm{kV}$ ) with increasing layers of plastic wrap at the end of the cone. Multiple readings were taken and averaged for each thickness. This was repeated at an optimal thickness for all available energies.

\section{Dose Reduction with and without Sleeve}

Finally, the printed sleeve was inserted into the cone and output was measured and compared with the open output. A $1 \mathrm{~mm}$ thick sleeve was designed in TinkerCad (Autodesk Inc) software and printed at a commercial printer. The wall thickness of 1 $\mathrm{mm}$ was the thinnest that could be reliably printed and still provide a robust structure. The initial print design was taped in place within the cone, shown in Figure 1. The reduction in dose for the low energy chamber was determined and compared to the ROF measured using the Farmer chamber.

\section{Results}

\section{Electron dose contribution}

The dose at the end of the two cones was measured for all available clinical energies using the Farmer chamber and the Exradin. The Relative Output Factors (ROF) were calculated, and the difference between the two chambers has been attributed to electron enhanced dose, see Table 2. The increase in dose measured by the Exradin chamber varied between 9.9 to $14.4 \%$.

Table 2

Measured ROFs of the $4 \mathrm{~cm}: 5 \mathrm{~cm}$ Cone using two chamber types, measured in-air at end of the cone.

\begin{tabular}{|c|c|c|c|}
\hline Energy/Filter & ROF Farmer & ROF Exradin & \%Difference \\
\hline Filter 1 & 1.1636 & 1.2855 & -10.5 \\
\hline Filter 2 & 1.0773 & 1.1838 & -9.9 \\
\hline Filter 3 & 1.0415 & 1.1703 & -12.4 \\
\hline Filter 4 & 1.0234 & 1.1704 & -14.4 \\
\hline
\end{tabular}

\section{Dose Reduction Using Plastic Wrap}

Filter 2 was used to measure relative dose with increasing thicknesses of plastic wrap applied to the end of the cone. The reduction in dose compared to no plastic layers is shown in Table 3 and was up to $3 \%$ for the maximum thickness measured.

Table 3: Increasing thicknesses of plastic wrap were applied to the end of the $4 \mathrm{~cm}$ cone and output was measured using the Exradin Chamber. 


\begin{tabular}{|lll|}
\hline Number of Plastic Layers & $\begin{array}{l}\text { Reading } \\
\text { (nC) }\end{array}$ & Reduction (\%) \\
\hline 0 & 132.9 & 0.0 \\
2 & 131.8 & 0.9 \\
6 & 131.2 & 1.3 \\
8 & 130.3 & 2.0 \\
\hline
\end{tabular}

The reduction in output at the end of the cone was measured for all other available energies using eight layers of plastic wrap for comparison. The reduction in dose is shown in Table 4.

Table 4

Reduction in measured dose using eight layers of plastic wrap for all other energies.

\begin{tabular}{|ll|}
\hline Energy/Filter & Reduction (\%) \\
\hline Filter 1 & 2.8 \\
Filter 3 & 6.1 \\
Filter 4 & 9.3 \\
\hline
\end{tabular}

\section{Dose Reduction with and without Sleeve}

The output at the end of the cone was measured with and without the 3D printed sleeve for the $4 \mathrm{~cm}$ cone. Measurements were made with the Exradin chamber and the reduction in dose was up to $15.2 \%$ as shown in Table 5 .

Table 5

ROF measured at the end of the cone with and without the inner sleeve (with Exradin).

\begin{tabular}{|llll|}
\hline Energy/Filter & ROF with Sleeve & ROF without Sleeve & $\begin{array}{l}\text { Reduction } \\
\text { (\%) }\end{array}$ \\
\hline Filter 1 & 1.1504 & 1.2855 & 10.5 \\
\hline Filter 2 & 1.0443 & 1.1838 & 11.8 \\
\hline Filter 3 & 1.0069 & 1.1703 & 14.0 \\
\hline Filter 4 & 0.9924 & 1.1704 & 15.2 \\
\hline
\end{tabular}

Table 6 compares the measured ROFs with the sleeve in place and the Farmer measured ROFs as a measure of electron dose reduction. The difference in Farmer to Sleeve was reduced to $<=3.3 \%$. 
Table 6

Comparison of the ROFs for the $4 \mathrm{~cm}$ and $5 \mathrm{~cm}$ cones under

different conditions and measurement chambers.

\begin{tabular}{|llll|}
\hline Energy/Filter & ROF Farmer & $\begin{array}{l}\text { ROF Sleeve } \\
\text { Exradin }\end{array}$ & $\begin{array}{l}\text { Difference } \\
\text { Farmer to Sleeve } \\
\text { (\%) }\end{array}$ \\
\hline Filter 1 & 1.1636 & 1.1504 & 1.1 \\
Filter 2 & 1.0773 & 1.0443 & 3.1 \\
Filter 3 & 1.0415 & 1.0069 & 3.3 \\
Filter 4 & 1.0234 & 0.9924 & 3.0 \\
\hline
\end{tabular}

\section{Discussion}

Initial measurements undertaken in Table 2 show the ratio of output between the $4 \mathrm{~cm}$ and $5 \mathrm{~cm}$ cone between two different chambers to estimate the electron enhancement for the $4 \mathrm{~cm}$ cone. The $5 \mathrm{~cm}$ cone is close-ended, and thus absorbs the electron enhancement contribution. In addition, the measurements performed using the Farmer reference chamber do not show the enhanced dose due to the wall thickness of the Farmer. The wall thickness for the Farmer chamber exceeds the recommended minimum thickness as listed Table 1 of TG61 ((Ma et al., 2001). Therefore, measured doses using the Farmer chamber are only due to the kilovoltage photons. Measurements made with a low-energy chamber, such as the Exradin, with a much thinner front window, will include electron dose. The ROFs shown in Table 2 estimate the electron dose contribution to be within $9.9-14.4 \%$ for the range of energies measured. This measurement agrees with other researchers estimates of up to 14\% (Hang et al., 2000).

The use of plastic wrap showed that there could be an appreciable dose reduction by up to $9 \%$. Plastic wrap could provide a reasonable solution as it is readily available. In this series of measurements, eight layers of wrap were used, but this does not remove all enhanced dose and further layers would be needed to eradicate the effect. In layering plastic wrap, it is difficult for the layers to be made uniformly and without wrinkles. The additional time taken in a busy clinic would be non-trivial to prepare this for all patients, as well as the need of consideration of re-using for the multiple fractions, or waste by making new for each fraction for each patient. Other commercially available thicker plastic sheeting $(200 \mu \mathrm{m})$ such as "Builders Film" maybe reasonable to use, and reuse, but have not been measured in this study.

The use of the internal sleeve showed a reduction in the dose delivered by up to approximately $15 \%$. This would indicate complete eradication of the electron dose to the measurement point. Table 6 compares the ROFs measured with the Farmer chamber, and the Exradin chamber, with and without the sleeve. The sleeved measurements are within 3.3\% of the Farmer chamber measurements. It is noted that for Filter 1, the ROF is within $1.1 \%$ of the Farmer chamber value, but the Farmer chamber is not recommended for this energy range.

One factor that has not been measured in this study was the reduction in field size by the inclusion of the sleeve. It is anticipated that film measurements would demonstrate some field size reduction, and penumbral broadening. Given these considerations, the clinic has decided to not include a sleeve for the smallest cone available. The $1 \mathrm{~cm}$ cone is used generally to treat inner canthus lesions. A $1 \mathrm{~mm}$ thick wall for the inner sleeve could potentially shrink the treatment size for full dose by up to $20 \%$. For the small number of fields using this cone, the plastic wrap would provide a preferable option.

In addition, taping the sleeve in place is non-ideal. Subsequent print design was refined to ensure a snug fit of the sleeve in place. The sleeve can be removed for cleaning as required or replaced should it degrade.

\section{Conclusion}

This work examined the use of a simple 3D printing option to remove the enhanced electron dose observed at the end of openended stainless-steel treatment cones. The sleeves provided a reduction in measured dose by up to $15 \%$, aligning the measured 
ROF closely to that from a Farmer type chamber. Plastic food wrap is also able to remove some of the dose but with handling impracticalities is less desirable in a busy clinical department.

\section{Declarations}

\section{Compliance with Ethical Standards:}

Ethical approval: This article does not contain any studies with human participants performed by the author.

Conflicts of Interest: The author has received a research grant from Wolf Medical.

Acknowledgements The author gratefully acknowledges Hans Feitz from Gamma Gurus for the use of his Exradin chamber.

\section{References}

1. Craft, D. F., Kry, S. F., Balter, P., Salehpour, M., Woodward, W., \& Howell, R. M. (2018). Material matters: Analysis of density uncertainty in 3D printing and its consequences for radiation oncology. Medical Physics, 45(4), 1614-1621.

https://doi.org/10.1002/mp.12839

2. Crowe, S. B., Charles, P. H., Cassim, N., Maxwell, S. K., Sylvander, S. R., Smith, J. G., \& Kairn, T. (2021). Predicting the required thickness of custom shielding materials in kilovoltage radiotherapy beams. Physica Medica, 81, 94-101.

https://doi.org/https://doi.org/10.1016/j.ejmp.2020.12.008

3. Hang, C., Lee, M., Kwan, K., \& Chan, D. (2000). Electron contamination from the lead cutout used in kilovoltage radiotherapy. Physics in Medicine and Biology, 145, 1-8.

4. Hill, R., Healy, B., Butler, D., Odgers, D., Gill, S., Lye, J., Gorjiara, T., Pope, D., \& Hill, B. (2018). Australasian recommendations for quality assurance in kilovoltage radiation therapy from the Kilovoltage Dosimetry Working Group of the Australasian College of Physical Scientists and Engineers in Medicine. Australasian Physical \& Engineering Sciences in Medicine, 41(4), 781-808. https://doi.org/10.1007/s13246-018-0692-1

5. Klevenhagen, S. C., D’Souza, D., \& Bonnefoux, I. (1991). Complications in low energy X-ray dosimetry caused by electron contamination. Physics in Medicine and Biology, 36(8), 1111-1116. https://doi.org/10.1088/0031-9155/36/8/006

6. Ma, C. M., Coffey, C. W., DeWerd, L. A., Liu, C., Nath, R., Seltzer, S. M., \& Seuntjens, J. P. (2001). AAPM protocol for 40-300 kV x-ray beam dosimetry in radiotherapy and radiobiology. Medical Physics, 28(6), 868-893. https://doi.org/10.1118/1.1374247

7. McGregor, S., Minni, J., \& Herold, D. (2015). Superficial radiation therapy for the treatment of nonmelanoma skin cancers. Journal of Clinical and Aesthetic Dermatology, 8(12), 12-14.

8. Perera, E., Gnaneswaran, N., Staines, C., Win, A. K., \& Sinclair, R. (2015). Incidence and prevalence of non-melanoma skin cancer in Australia: A systematic review. Australasian Journal of Dermatology, 56(4), 258-267.

https://doi.org/10.1111/ajd.12282

9. Polyactic Acid. (2015). Polymer Database. https://polymerdatabase.com/polymers/polylacticacid.html\%0D\%0A

10. Polyvinyl Chloride PVC: Properties, Benefits \& Applications. (2019). British Plastics Federations.

https://www.bpf.co.uk/plastipedia/polymers/pvc.aspx\%0Ahttps://www.bpf.co.uk/plastipedia/polymers/pvc.aspx\#Physical Properties\%0Ahttps://www.bpf.co.uk/plastipedia/polymers/pvc.aspx

11. Van der Walt, M., Crabtree, T., \& Albantow, C. (2019). PLA as a suitable 3D printing thermoplastic for use in external beam radiotherapy. Australasian Physical and Engineering Sciences in Medicine, 42(4), 1165-1176.

https://doi.org/10.1007/s13246-019-00818-6

\section{Figures}



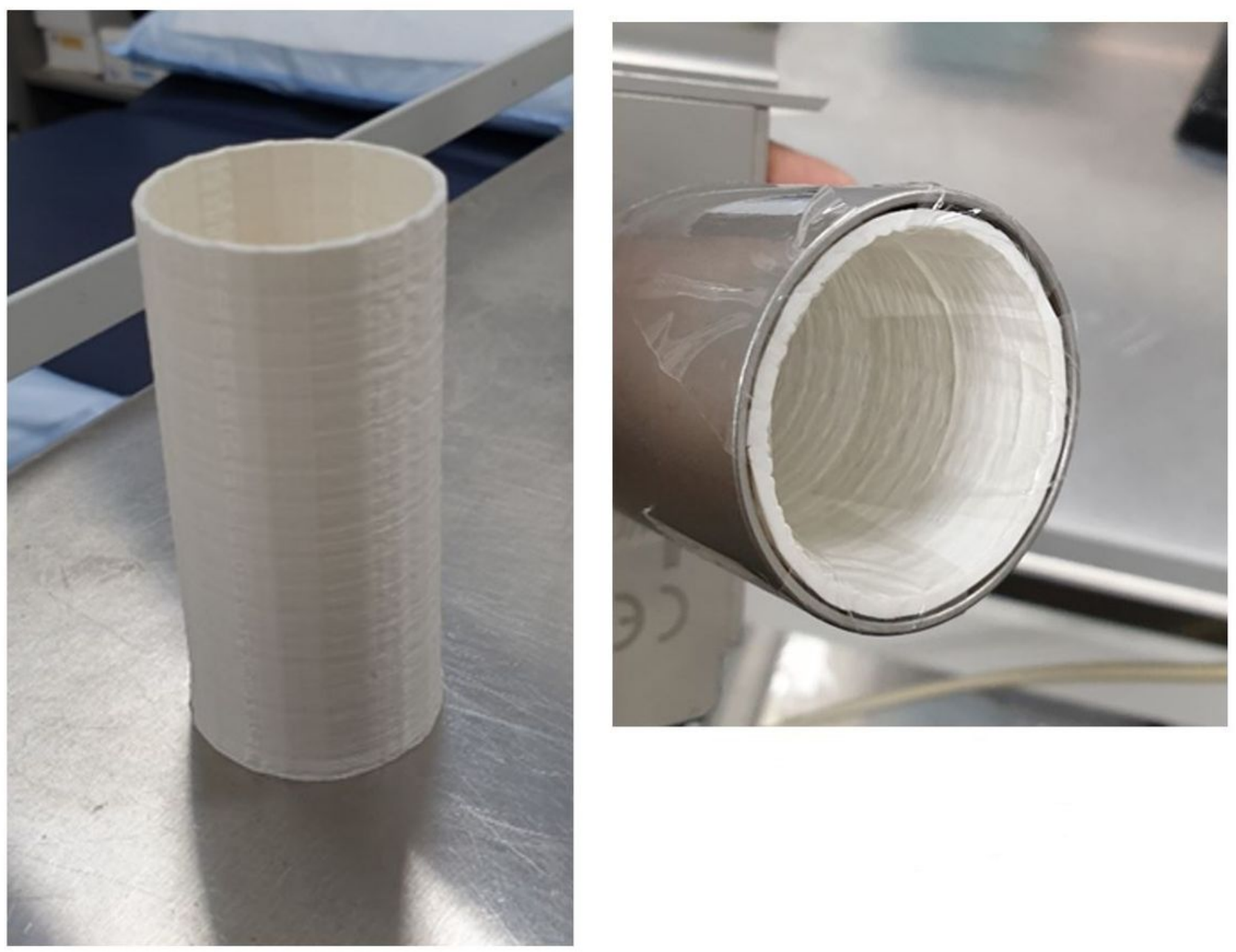

Figure 1

(a) Initial trial sleeve manufactured at $1 \mathrm{~mm}$ thickness and $15 \mathrm{~cm}$ long using PLA. (b) Sleeve placed within cone and secured by tape. 